\title{
Genome-wide identification, characterization, and evolutionary analysis of flowering genes in radish (Raphanus sativus L.)
}

Jinglei Wang, Yang Qiu, Feng Cheng, Xiaohua Chen, Xiaohui Zhang, Haiping Wang, Jiangping Song, Mengmeng Duan, Haohui Yang and Xixiang Li*

\begin{abstract}
Background: Radish (Raphanus sativus L.) belongs to the family Brassicaceae, and is an economically important root crop grown worldwide. Flowering is necessary for plant propagation, but it is also an important agronomic trait influencing $R$. sativus fleshy taproot yield and quality in the case of an imbalance between vegetative and reproductive growth. There is currently a lack of detailed information regarding the pathways regulating the flowering genes or their evolution in $R$. sativus. The release of the $R$. sativus genome sequence provides an opportunity to identify and characterize the flowering genes using a comparative genomics approach.

Results: We identified 254 R. sativus flowering genes based on sequence similarities and analyses of syntenic regions. The genes were unevenly distributed on the various chromosomes. Furthermore, we discovered the existence of $R$. sativus core function genes in the flowering regulatory network, which revealed that basic flowering pathways are relatively conserved between Arabidopsis thaliana and R. sativus. Additional comparisons with Brassica oleracea and Brassica rapa indicated that the retained flowering genes differed among species after genome triplication events. The $R$. sativus flowering genes were preferentially retained, especially those associated with gibberellin signaling and metabolism. Moreover, analyses of selection pressures suggested that the genes in vernalization and autonomous pathways were more variable than the genes in other $R$. sativus flowering pathways.

Conclusions: Our results revealed that the core flowering genes are conserved between $R$. sativus and $A$. thaliana to a certain extent. Moreover, the copy number variation and functional differentiation of the homologous genes in $R$. sativus increased the complexity of the flowering regulatory networks after genome polyploidization. Our study provides an integrated framework for the $R$. sativus flowering pathways and insights into the evolutionary relationships between $R$. sativus flowering genes and the genes from $A$. thaliana and close relatives.
\end{abstract}

Keywords: Raphanus sativus L., Genome-wide, Flowering genes, Regulatory pathway networks, Evolution

\footnotetext{
* Correspondence: lixixiang@caas.cn

Institute of Vegetables and Flowers, Chinese Academy of Agricultural

Sciences, Key Laboratory of Biology and Genetic Improvement of

Horticultural Crops, Ministry of Agriculture, Beijing 100081, China
} 


\section{Background}

Flowering is a necessary part of plant propagation, and the process from bolting to blooming is a crucial period for the transition of Brassicaceae plants from vegetative to reproductive growth. Comprehensively characterizing the regulatory mechanisms underlying bolting and blooming may enable researchers to influence the balance between vegetative and reproductive growth, which may ultimately affect the yield and quality of Brassicaceae crops.

Approximately 174 genes are believed to regulate flowering in the model plant Arabidopsis thaliana, which are involved in six major pathways [i.e., vernalization, photoperiod and circadian clock, ambient temperature, gibberellin (GA), age, and autonomous pathways] influencing the bolting or blooming process [1]. Although different genes are responsible for different internal and environmentally mediated flowering pathways, the different pathways appear coordinated primarily by a few floral integrator genes, including FLOWERING LOCUS T (FT), LEAFY (LFY), and SUPPRESSOR OF OVEREXPRESSION OF CONSTANS1 (SOC1) [1]. The recent completion of genome sequences and the development of novel computational analysis techniques have enabled the genome-wide identification and characterization of flowering genes in economically important plants. For example, 900 and 275 putative flowering genes in Triticum aestivum and Hordeum vulgare [2] respectively, 96, 98 and 304 flowering gene homologs in Lotus corniculatus var. japonicus, Medicago truncatula and Glycine max [3] separately, have been identified. The genes regulating bolting and flowering vary among different crops.

Raphanus sativus is a member of the family Brassicaceae, and is cultivated worldwide. It has recently undergone tetraploidization events ( $\alpha$ and $\beta$ ) with $A$. thaliana, B. oleracea and $B$. rapa, as well as a whole genome triplication with $B$. oleracea and $B$. rapa following their divergence from $A$. thaliana $[4,5]$. $R$. sativus has similar flowering habits to $A$. thaliana, $B$. oleracea, and $B$. rapa, with a highly variable flowering time and diverse responses to temperature and/or day length. Fifty flowering miRNAs targeting 154 transcripts [6], and 95 flowering genes differentially expressed between the vegetative and reproductive stages [7], have been identified in $R$. sativus. Additionally, 290 flowering genes have been detected in the $R$. sativus genome [8]. However, little attention has been paid to their characteristics or evolution of the genes in different pathways regulating $R$. sativus flowering.

In this study, we systematically identified flowering genes in the $R$. sativus genome, and uncovered new details regarding the presence or absence of these genes. We also investigated dominant pathways as well as the evolutionary relationships and expression profiles among the flowering genes. Our findings may provide useful information and enlightenment for breeders to improve bolting and flowering in R. sativus and other Brassicaceae crops.

\section{Methods}

\section{Data resources}

Details regarding the annotated $A$. thaliana genome were downloaded from the TAIR10 website (http://www.arabidopsis.org) [9]. The R. sativus, B. rapa and B. oleracea genome assembly and gene annotation data were downloaded from the BRAD database (http://brassicadb.org/brad/) [10]. Genomic data for Vitis vinifera, Populus trichocarpa, Carica papaya, and Thellungiella salsuginea were obtained from the Genoscope (http://www.genoscope.cns.fr/spip/), JGI Genome Portal (http:/genome.jgi.doe.gov/), plantGDB (http:/www.plantgdb.org/), and Omicslab (http://omicslab.genetics.ac.cn/resources.php) databases, respectively. $R$. sativus RNA-seq data [11] are available at EMBL/NCBI/SRA (PRJNA413464). The core eukaryotic genes were downloaded from CEGMA (http://korflab.ucdavis.edu/datasets/cegma/) [12].

\section{Identification of flowering gene homologs in Raphanus sativus}

We identified homologous genes using a combination of similarity- and synteny-based approaches. In the similaritybased approach, BLASTP searches were conducted against $R$. sativus protein sequences using the following conditions: E-value $<1$ e- 20 , identity $>50 \%$, coverage $>60 \%$, and match length $>60$ amino acids. In the synteny-based approach, SynOrths software (http://brassicadb.org/brad/downloadOverview.php), which determines whether two genes are a conserved syntenic pair based on sequence similarities and homologies of their flanking genes, was used to identify syntenic $A$. thaliana and $R$. sativus genes [13]. We further defined homologous relationships among the similar and syntenic genes. Multiple gene sequences were aligned using CLUSTALW [14], and phylogenetic trees were constructed using the neighbor-joining method of the MEGA 6.0 software (1000 bootstrap replicates) [15]. Putative homologous genes were manually checked on the phylogenetic trees.

\section{Localization of flowering genes in the Raphanus sativus genome}

To construct physical maps indicating the distribution of flowering genes, genome localization details for the predicted $R$. sativus flowering genes were collected from the annotation information. The MG2C (http://mg2c.iask.in/mg2c_v2.0/) program was used to visualize the putative flowering genes on nine pseudo-molecular chromosomes [16].

Flowering gene expression analysis based on RNA-seq data We analyzed the transcriptomes of six different tissues (i.e., flowers, siliques, leaves, stem, callus, and roots) collected from $R$. sativus inbred line XYB36-2 [11]. Transcript abundance was calculated according to the FPKM method (fragments per kilobase of exon per million mapped reads) using Cufflinks [17] and TopHat2 [18]. Heatmaps were generated with the $\mathrm{R}$ package pheatmap [19]. 


\section{Non-synonymous/synonymous substitution ratios of flowering gene pairs between Arabidopsis thaliana and Raphanus sativus}

The non-synonymous/synonymous substitution ratio (Ka/ $\mathrm{Ks}$ ) of homologous gene pairs is related to the evolutionary selection patterns of the corresponding genome. In the calculation of $\mathrm{Ka} / \mathrm{Ks}$, The full length of amino acid sequences of the $R$. sativus and A. thaliana flowering genes underwent pairwise alignments using MUSCLE [20] firstly. Then, The aligned amino acid sequences were translated into the corresponding nucleotides coding sequences using PERL scripts. Finally, the translated nucleotides coding sequences were used as input files in computing $\mathrm{Ka} / \mathrm{Ks}$ values using
Li-Wu-Luo model [21] integrated in KaKs_Calculator2 software [22]. All variable sites of the alignment pairs were used in the $\mathrm{Ka} / \mathrm{Ks}$ calculation. To detect selection pressures, $\mathrm{Ka} / \mathrm{Ks}$ ratios greater than 1 , less than 1 , and equal to 1 were considered to represent positive selection, negative or stabilizing selection, and neutral selection, respectively.

\section{Results}

\section{Identification of Raphanus sativus flowering genes}

There are 174 genes, including $24 \mu$-RNA genes, with known functions affecting $A$. thaliana flowering time [1]. We focused on the 160 protein-coding genes to identify homologous $R$. sativus flowering genes. We identified 254

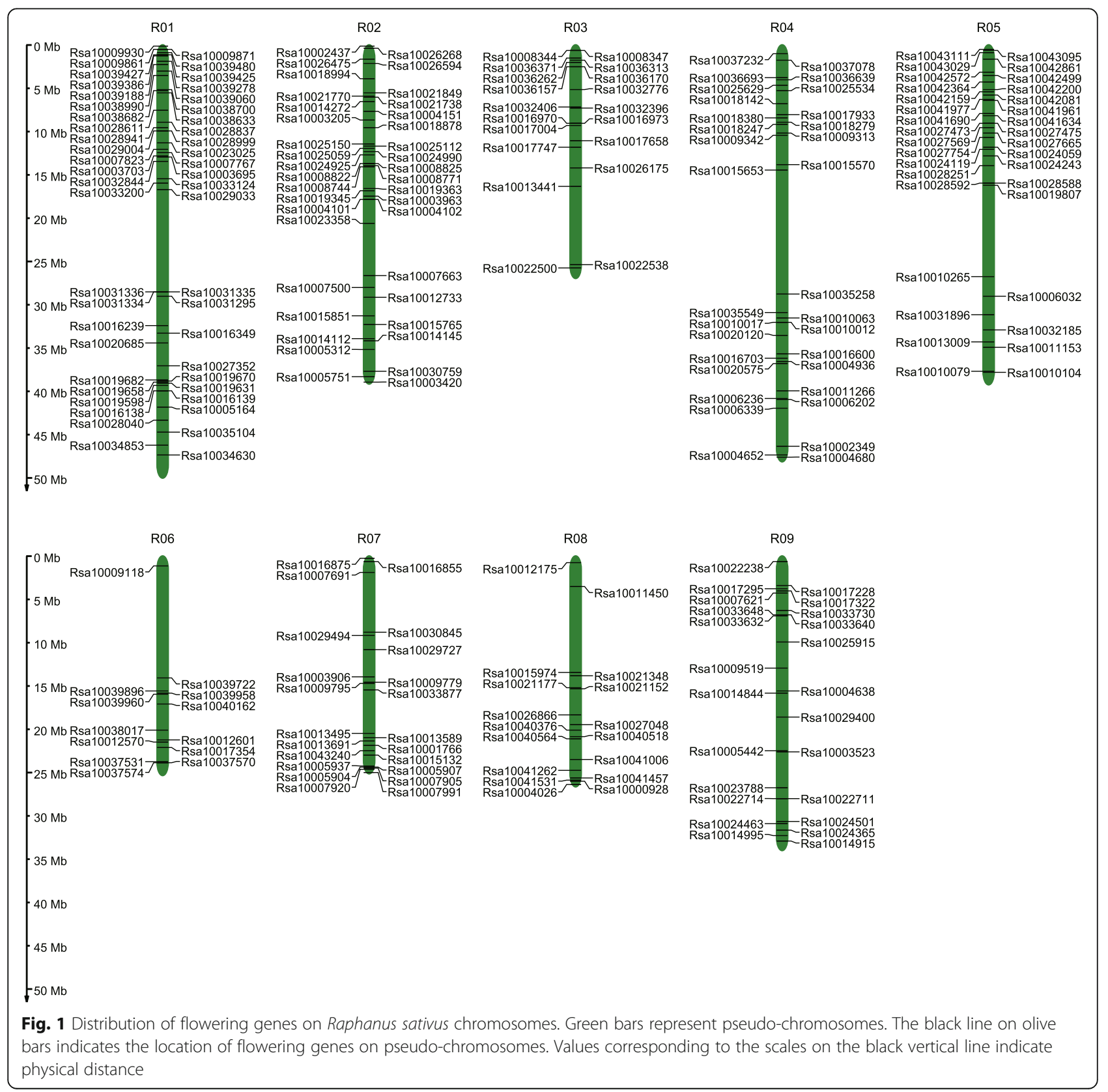


$R$. sativus flowering genes (Additional file 1), and determined that most of the $A$. thaliana flowering genes have putative $R$. sativus homologs (139 out of 160). Homologs in the $R$. sativus genome were lacking for 21 genes, and most of these genes (15 of 21) have functionally redundant effects on flowering (Additional file 2). Interestingly, all of the lost genes (8 genes) which belonging to photoperiod pathway, circadian clock, and light signaling genes set have function redundant genes retained in $R$. sativus.

\section{Distribution of Raphanus sativus flowering genes on pseudo-molecular chromosomes}

We mapped $247 R$. sativus flowering genes onto pseudomolecular chromosomes, while the remaining seven genes were assigned to unanchored scaffolds (Fig. 1). The distribution of these genes was uneven, with 48 genes localized on chromosome 1, representing $19.43 \%$ of the flowering genes. Only 14 flowering genes $(5.66 \%)$ were detected on chromosome 6 , with most located on the bottom half.

\section{Comparison of flowering genes from Arabidopsis thaliana,} Brassica oleracea, Brassica rapa, and Raphanus sativus

As Brassicaceae species, $B$. oleracea and $B$. rapa have been sequenced and studied in-depth [23-25]. We used the abovementioned method to identify homologous flowering genes in B. oleracea and B. rapa. The fewest number of flowering genes were identified for $R$. sativus (Fig. 2 and Additional file 3), even though it had the second most annotated genes $(43,240)$ [11], which is between $B$. oleracea $(45,758)$ [23] and B. rapa $(41,174)$ [24]. There were no significant differences in the number of identified flowering genes among $R$. sativus, B. oleracea and B. rapa (Chisquared test $=2.3224, P$ value $=0.1275$ ) .

Except for four genes that could not be categorized, the putative $R$. sativus, B. oleracea, and B. rapa flowering genes were classified into the following four gene sets: GA signaling and metabolism; vernalization and autonomous pathways; photoperiod pathway, circadian clock, and light signaling; and meristem response and development,

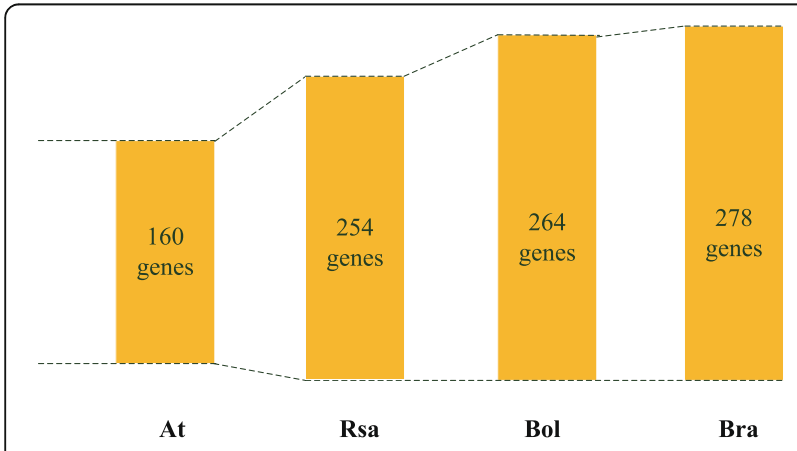

Fig. 2 Flowering genes in different species. At, Arabidopsis thaliana; Rsa, Raphanus sativus; Bol, Brassica oleracea; Bra, Brassica rapa. Numbers in boxes correspond to the number of genes according to the classification of $A$. thaliana genes [1] (Table 1 and Additional file 3). Most of the genes belonged to the photoperiod pathway, circadian clock, and light signaling gene set. There was little difference in the numbers of $R$. sativus, B. oleracea, and B. rapa genes associated with GA signaling and metabolism.

\section{Dominant pathways and key families of Raphanus sativus flowering genes \\ Photoperiod pathway, circadian clock, and light signaling}

Plants can sense day length changes and use them to control the onset of flowering. We identified $101 R$. sativus genes that were homologous to $58 \mathrm{~A}$. thaliana genes of photoperiod pathway, circadian clock, and light signaling (Additional file 1).

CONSTANS $(\mathrm{CO})$, which acts as a point of integration of the internal circadian clock and the external day-night cycles, plays a central role in photoperiodic flowering control of plants [26]. In R. sativus, one copy was identified to be homologous of $C O$. Significantly, the genes involved in circadian clock including CCA1, LHY, TOC1, GI, CDF1 and $L K P 2$ [27-30] were identified in $R$. sativus. LKP2 belongs to a family of F-box proteins, which also include ZTL and FKF1 [27]. ZTL and FKF1 are both lost and LKP2 have three tightly linked copies in $R$. sativus, that are similar to that of $B$. rapa [25], suggesting the lose of $Z T L$ and FKF1 and the local triplication event of LKP2 may have taken place in the common ancestor of $R$. sativus and B. rapa. In addition, CRY1, CRY2, PHYA, PHYB, $P H Y C$, and $P H Y E$ being implicated in plant light signaling pathways [31] were identified in $R$. sativus.

\section{Vernalization and autonomous pathways}

Many plants growing in temperate climates require vernalization (i.e., prolonged exposure to low temperatures), which involves the silencing of $F L C$, to initiate or accelerate the flowering process [32]. Similar to the genes of the vernalization pathway, genes in the autonomous pathway

Table 1 Number of flowering genes in Arabidopsis thaliana, Raphanus sativus, Brassica oleracea, and Brassica rapa

\begin{tabular}{lllll}
\hline $\begin{array}{l}\text { Flowering pathways } \\
\text { and their gene sets }\end{array}$ & A. thaliana & R. sativus & B. oleracea & B. rapa \\
\hline $\begin{array}{l}\text { Photoperiod pathway, } \\
\text { circadian clock, } \\
\text { light signaling }\end{array}$ & 67 & 101 & 107 & 114 \\
$\begin{array}{l}\text { Gibberellin signaling } \\
\text { and metabolism }\end{array}$ & 21 & 35 & 33 & 34 \\
$\begin{array}{l}\text { Vernalization and } \\
\text { autonomous pathways }\end{array}$ & 49 & 72 & 72 & 81 \\
$\begin{array}{l}\text { Meristem response } \\
\text { and development }\end{array}$ & 19 & 38 & 44 & 42 \\
Other & 4 & 8 & 8 & 7 \\
\begin{tabular}{l} 
Total \\
\hline
\end{tabular} & 160 & 254 & 264 & 278 \\
\hline
\end{tabular}


normally indirectly promote flowering by repressing the floral repressor $F L C$ [33]. FLC, which is a MADS-box gene, is the major flowering repressor in the vernalization pathway [34]. Three FLC homologs were identified in $R$. sativus. As expected, most of vernalization-response genes including VIN3, VRN1, VRN2, FRI were also identified in this study [35-38]. Furthermore, in the FLC-independent vernalization pathway, prolonged exposure to cold conditions can elevate AGL19 and AGL24 expression levels, which can activate $L F Y$ and $A P 1$ expression and eventually leads to flowering [39]. The $R$. sativus contained three copies of AGL19 and two copies of AGL24.

Moreover, we also identified homologous genes in autonomous pathway, including $L D, F C A, F Y, F P A, F V E$, $F L D$, and $F L K$. All autonomous genes are indirectly involved in inducing early flowering through the repression of $F L C$ [40].

\section{Gibberellin signaling and metabolism}

The initiation of flowering in A. thaliana under noninductive short-day conditions can be promoted by GA. There are 35 genes likely related to the GA pathway in $R$. sativus. The GA pathway genes are classified as those associated with GA biosynthesis (e.g., GA2ox, GA3ox, GA20ox [41]) and those acting as key signal transduction factors (e.g., SLY1, RGA, and GID1 [42]). Except for GAI, homologs of the all GA biosynthesis genes and transduction factors were retained in $R$. sativus. The GAI and $R G A$ genes are members of the DELLA family, which repress GA-induced vegetative growth and floral initiation [43].

\section{Meristem response and development}

The onset of flowering is largely dependent on the expression of a relatively small number of central floral pathway factors that integrate signals from several related pathways

a

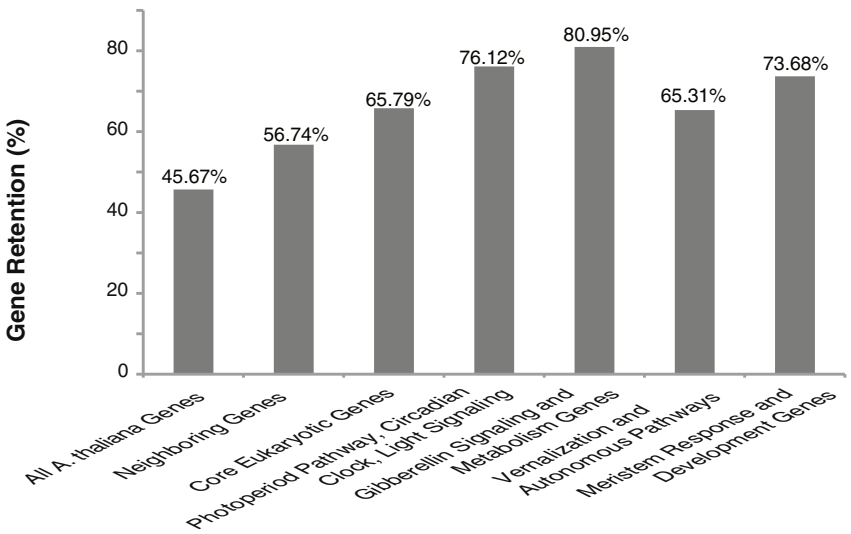

b

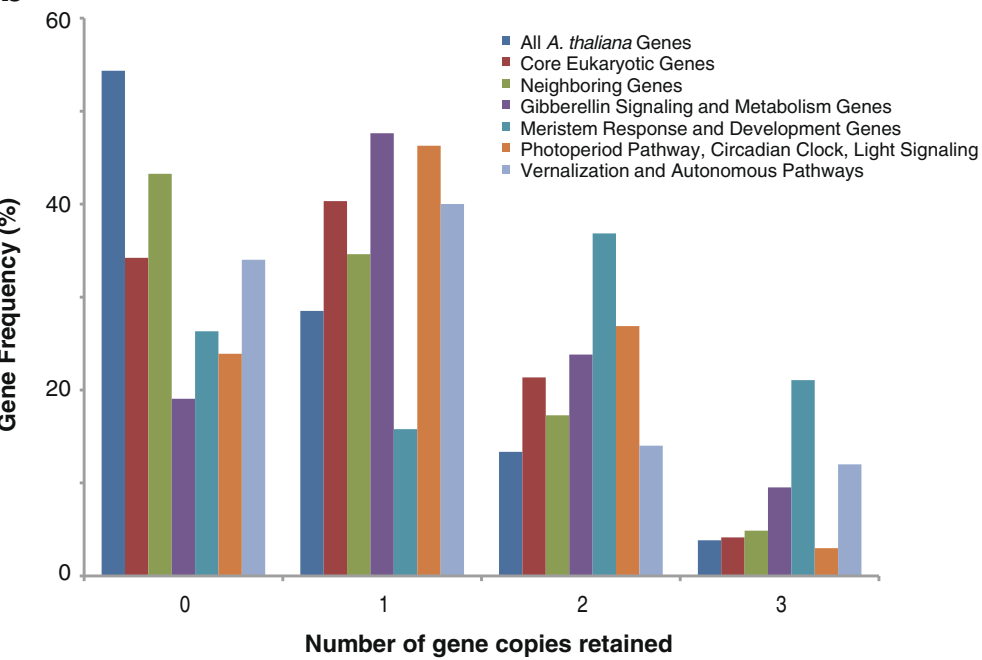

Fig. 3 Number of flowering gene homologs retained as syntenic genes in Raphanus sativus. The retained homologs among the four gene sets of flowering genes and their immediate neighbors, all A. thaliana genes, and 459 core eukaryotic genes were included in the analysis. a Ratios of the retained genes in different gene sets. $\mathbf{b}$ Number of retained homologous genes in different gene sets 
during floral transitions [44]. In our study, $38 R$. sativus genes were identified as floral integrators or were associated with the flower meristem, including SOC1, FT, AP1, $L F Y$, and FD. Jung et al. (2016) did not detect $R$. sativus $L F Y$ through transcriptomic analysis [45], while, we detect two copies, although they both little expressed, which indicated $L F Y$ may expressed in specific periods and tissues.

\section{Differential retention of flowering genes in various species}

The gene dosage hypothesis predicts that genes whose products are dose-sensitive or interact with other proteins or in networks are over-retained, [46]. We compared the retention of genes from each of the above-mentioned four gene sets and three other gene sets: all $A$. thaliana genes, 2780 genes flanking the flowering genes (10 on either side), and 459 core eukaryotic genes. Overall, $80.95 \%$ of the GA signaling and metabolism genes, $76.12 \%$ of the photoperiod pathway, circadian clock, and light signaling genes, 73.68\% of the meristem response and development genes, and $65.31 \%$ of the vernalization and autonomous pathway genes were retained as syntenic genes. In contrast, $65.79 \%$ of the core eukaryotic genes, $56.74 \%$ of the neighboring genes, and $45.67 \%$ of all $A$. thaliana genes were retained as syntenic genes (Chi-squared test $=232.5112, P<0.001)$ (Fig. 3a). Most (57.89\%) of the meristem response and development genes were retained as two or three copies (Fig. 3b).

\section{Selection pressure on flowering pathway gene sets}

The $\mathrm{Ka} / \mathrm{Ks}$ ratios for homologous gene pairs were estimated to determine the direction and magnitude of natural selection acting on the $R$. sativus flowering genes. The mean $\mathrm{Ka} / \mathrm{Ks}$ ratios of different flowering gene sets ranged from 0.18 to 0.25 (Fig. 4 and Additional file 4), suggesting that negative selection had acted against extreme polymorphic variants in flowering genes. In particular, genes of the vernalization and autonomous pathways appear to have been subjected to less negative selection pressures than the genes from other pathways.

\section{Raphanus sativus Flowering gene expression analysis}

To characterize the divergence in the expression patterns of homologous genes and confirm their involvement in flowering, we analyzed the expression of the putative $R$. sativus flowering genes. By comparing transcript abundances in roots, stem, leaves, flowers, siliques, and callus, we determined that the expression of 16 putative flowering genes was undetectable in all tissues (Additional file 5). Furthermore, transcripts for most of the expressed genes (183 of 254) accumulated in flowers (Additional file 5), with seven genes that were preferentially or specifically expressed in flowers. Four of these seven genes (i.e., LMI1, SPL4, SPL5, and TFL1) were related to meristem response and development. Besides, it was found that COP1 and VIL1, only have one copy

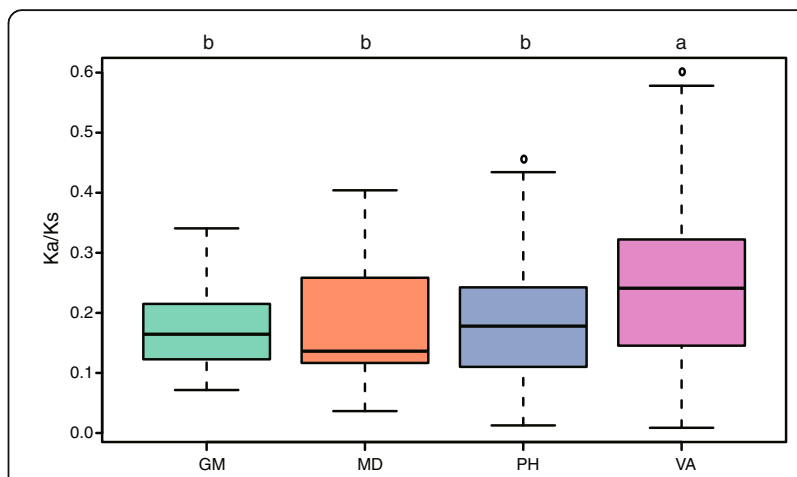

Fig. 4 Direction and magnitude of natural selection acting on different flowering gene sets. Quantile boxplots $(0.25,0.75)$ show the distribution of $\mathrm{Ka} / \mathrm{Ks}$ values for homologous gene pairs. The horizontal bar in each box indicates the median value. The upper and lower bars correspond to the upper and lower adjacent values 1.5-times outside the inter-quartile range. Outliers are plotted as discrete dots. Different letters indicate significantly different values $(P<0.01)$ as measured by Scheffé's test. GM, gibberellin signaling and metabolism gene set; $\mathrm{MD}$, meristem response and development gene set; $\mathrm{PH}$, photoperiod pathway, circadian clock, and light signaling gene set; VA, vernalization and autonomous pathways gene set

in $R$. sativusand not expressed in all tissues. It seems that these genes have lost function in $R$. sativus.

Duplicated genes can undergo non-functionalization, neo-functionalization, or sub-functionalization [47]. We chose flowering genes with more than three copies in the $R$. sativus genome to analyze the divergence of the homologous gene expression patterns. Although some genes exhibited similar expression patterns, we also observed considerable differences, suggesting that some homologs are functionally similar, while others are functionally diverse (Fig. 5).

\section{Discussion}

In contrast to the phenotypic effects of vernalization, photoperiod, and GA on $R$. sativus flowering, which have been well studied [48], little is known about the mechanisms mediating the effects. To address this deficiency, we used a bioinformatics approach to analyze the $R$. sativus genes potentially involved in flowering. Based on studies of $A$. thaliana flowering genes, we identified 254 putative $R$. sativus flowering-like genes through a genome-wide comparative analysis. The number of flowering genes in $R$. sativus was slightly less than that in B. oleracea and B. rapa, which is reasonable considering the genome sizes of the three species [23, 24]. While, homologs for $21 \mathrm{~A}$. thaliana flowering genes were not detected in the $R$. sativus genome and many of them have function redundant genes retained in $R$. sativus. The loss of these functionally redundant genes might be due to gene dosage imbalances $[49,50]$. However, exactly why certain functionally redundant genes are lost during evolution is unclear. The lost flowering-related genes likely do not affect the core flowering pathways, considering $R$. sativus 


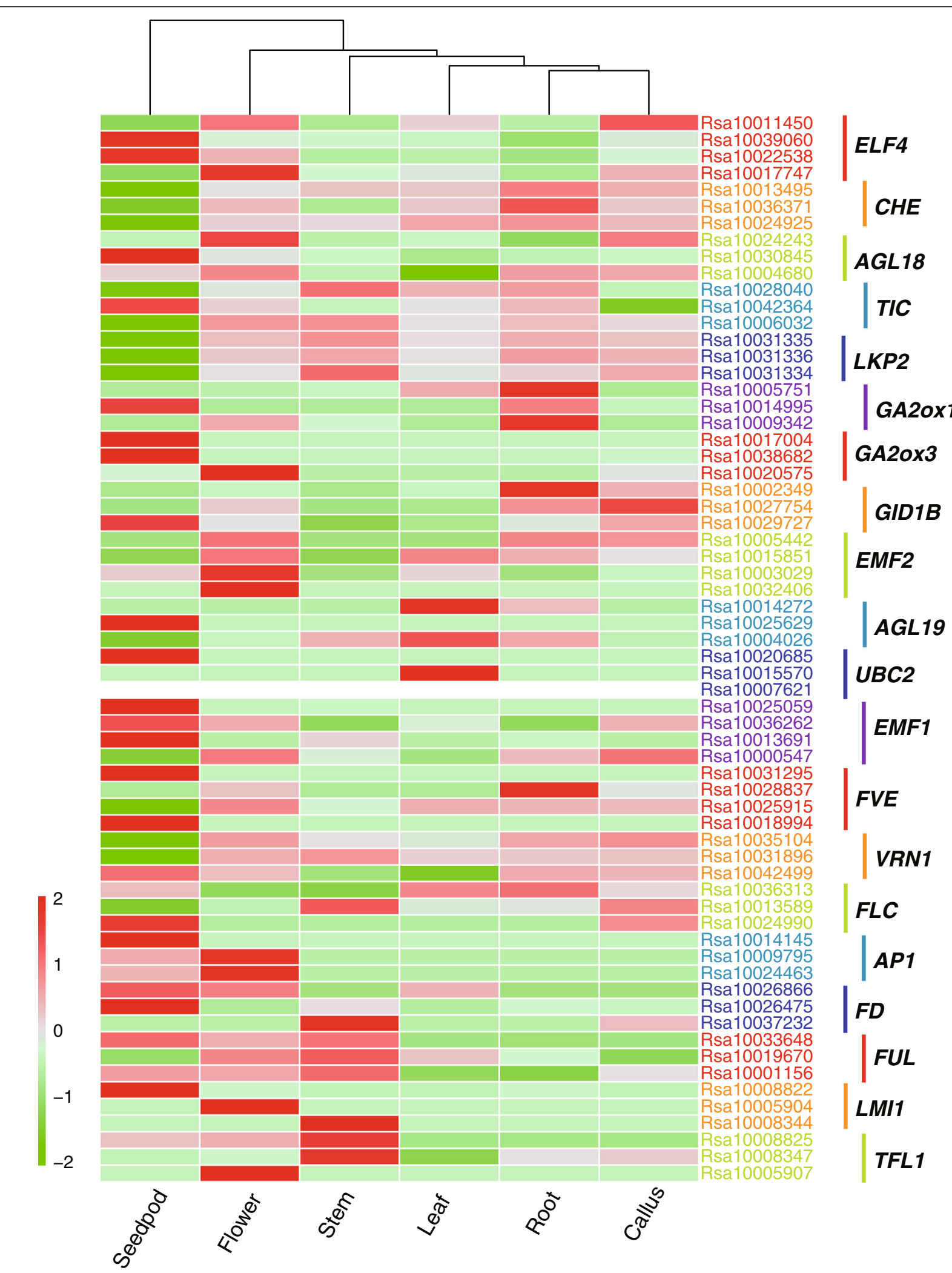

Fig. 5 Heatmap of Raphanus sativus flowering gene expression profiles. The analyzed tissues are indicated at the bottom of each column. The $R$. sativus gene codes to the right of the expression bar in the same color are homologs of the corresponding genes indicated on the right side with a colored line. The color scale bar at the bottom left of the figure represents log2 transformed FPKM values 
can still receive various endogenous and environmental cues that facilitate flowering.

Whole genome duplications and triplications are typically followed by a considerable loss of genes. However, the gene dosage hypothesis assumes that genes whose products participate in macromolecular complexes, signaling networks, or transcription in a dose-sensitive manner are over-retained, because an imbalance associated with the loss of one member of a complex or network is likely to decrease fitness [4951]. Many B. rapa circadian clock genes have exhibited preferential retention [25]. However, in this study, we observed that genes related to GA signaling and metabolism were preferentially retained over genes from other pathways. Additionally, genes from the photoperiod pathway, circadian clock, and light signaling gene sets or meristem response and development gene sets were also preferentially retained.

The results of our study suggest that basic flowering pathways are likely relatively conserved between $A$. thaliana and $R$. sativus. Three $R$. sativus FLC homologs were identified in this study, which is consistent with the findings of a transcript-level analysis by Yi et al. (2014), and their functions in transgenic $A$. thaliana have been examined [52]. Jung et al. (2016) also did not detect $R$. sativus FRI homologue through RNA-seq, while we identified two copies and both expressed [45]. The existence and expression of $F L C$ and FRI in $R$. sativus indicate that the FLC/FRI mode of action on vernalization is conserved, as are the components of the autonomous pathway [53]. Previous study reported that the genes in the vernalization pathway are not conserved between dicotyledonous and monocotyledonous plant species [2, 54]. Based on the $\mathrm{Ka} / \mathrm{Ks}$ ratios, we determined that the sequences of genes related to vernalization pathway were more variable than that of other pathways between $R$. sativus and A. thali$a n a$, which indicated vernalization gene sequences also exhibit great sequence diversity among dicotyledon plants. The variation of the vernalization gene sequences may contribute to the rapidly evolutionary capacity in changing thermal requirement to flowering in R. sativus [55].

Two $F T$ homologs and one copy of $C O$ were identified in $R$. sativus. This suggests a CO-FT module exists in $R$. sativus, which implies the photoperiod pathway control over flowering evolutionarily conserved in $R$. sativus and A. thaliana to a certain extent. In the dark, $\mathrm{CO}$ would be efficiently ubiquitinated by the COP1 E3 ligase complex and degraded, which contributed to late flowering in short days [56, 57]. The lost function of COP1 may suggest that CO would not be degraded in dark in $R$. sativus, which seems to be the reason for that $R$. sativus can flower in both short and long day.

\section{Conclusions}

We identified 254 putative flowering genes during a comparative genome analysis, and classified them into four flowering regulatory pathway gene sets in $R$. sativus. We also comprehensively analyzed the loss, presence, and variation of different pathway genes as well as the expression patterns of the flowering genes in R. sativus. Our results reveal that the flowering regulatory network is conserved between $R$. sativus and A. thaliana to a certain degree. The flowering-related genes were preferentially retained, especially those associated with GA signaling and metabolism. Furthermore, most of the R. sativus flowering genes lost during evolution were functionally redundant, possibly because of gene dosage imbalances. Moreover, analysis of selection pressures indicated that the vernalization and autonomous pathway genes are the most variable in $R$. sativus. Besides, The function loss of COP1 seems that photoperiod pathway can promote flowering in both short and long day in R.sativus.

In summary, our results further systematic and comprehensive understanding of the flowering regulatory molecular networks that evolved after a whole genome triplication event in $R$. sativus, which will be beneficial for breeders aiming to improve and regulate these processes in $R$. sativus and other Brassicaceae species.

\section{Additional files}

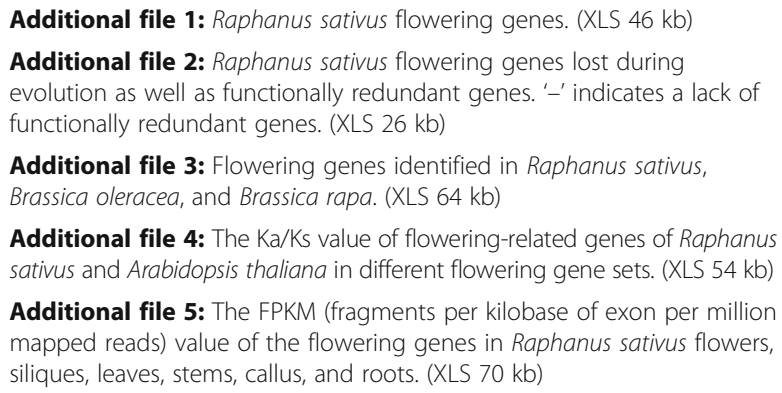

Additional file 3: Flowering genes identified in Raphanus sativus, Brassica oleracea, and Brassica rapa. (XLS 64 kb)

Additional file 4: The $\mathrm{Ka} / \mathrm{Ks}$ value of flowering-related genes of Raphanus sativus and Arabidopsis thaliana in different flowering gene sets. (XLS 54 kb)

Additional file 5: The FPKM (fragments per kilobase of exon per million mapped reads) value of the flowering genes in Raphanus sativus flowers, siliques, leaves, stems, callus, and roots. (XLS $70 \mathrm{~kb}$ )

\section{Acknowledgements}

Not applicable.

\section{Funding}

This work was supported by grants from the National Key Research and Development Plan of the Ministry of Science and Technology of the People's Republic of China (2013BAD01B04, 2016YFD0100204-02), 863 program (2012AA021801-04), and the Science and Technology Innovation Program of the Chinese Academy of Agricultural Sciences (CAAS-XTCX2016017, CAAS-XTCX2016016-4-4, CAAS-XTCX2016001-5-3).

\section{Availability of data and materials}

All data generated or analyzed during this study are included in this article and its supplementary information files. The raw reads of RNA-seq have been deposited in the NCBI Sequence Read Archive (SRA) database (BioProject PRJNA413464). The $R$. sativus genome data can be download from BRAD database (http://brassicadb.org/brad/).

\section{Authors' contributions}

$\mathrm{XL}$ conceived the project and revised the manuscript. JW designed and performed experiments, analyzed the data and wrote the manuscript. YQ, FC, XC, XZ, HW, JS, MD, and HY participated in experiments and analyzed the data. All authors read and approved the final manuscript. 


\section{Ethics approval and consent to participate}

Not applicable.

\section{Consent for publication}

Not applicable.

\section{Competing interests}

The authors declare that they have no competing interests.

\section{Publisher's Note}

Springer Nature remains neutral with regard to jurisdictional claims in published maps and institutional affiliations.

Received: 8 August 2016 Accepted: 11 December 2017

Published online: 19 December 2017

\section{References}

1. Fornara F, de Montaigu A, Coupland G. SnapShot: control of flowering in Arabidopsis. Cell. 2010;141:550-550.

2. Peng FY, Hu Z, Yang RC. Genome-wide comparative analysis of floweringrelated genes in Arabidopsis, wheat, and barley. Int J Plant Prod. 2015: 874361-1.

3. Kim MY, Kang YJ, Lee T, Lee SH. Divergence of flowering-related genes in three legume species. Plant Genome. 2013;6(3)

4. Bowers JE, Chapman BA, Rong JK, Paterson AH. Unravelling angiosperm genome evolution by phylogenetic analysis of chromosomal duplication events. Nature. 2003;422(6930):433-8.

5. Town CD, Cheung F, Maiti R, Crabtree J, Haas BJ, Wortman JR, Hine EE, Althoff R, Arbogast TS, Tallon LJ, et al. Comparative genomics of Brassica oleracea and Arabidopsis thaliana reveal gene loss, fragmentation, and dispersal after polyploidy. Plant Cell. 2006;18(6):1348-59.

6. Nie S, Xu L, Wang Y, Huang D, Muleke EM, Sun X, Wang R, Xie Y, Gong Y, Liu L. Identification of bolting-related microRNAs and their targets reveals complex miRNA-mediated flowering-time regulatory networks in radish (Raphanus sativus L). Sci Rep 2015:14034-4

7. Nie S, Li C, Wang Y, Xu L, Muleke E, Tang M, Sun X, Liu L. Transcriptomic analysis identifies differentially expressed genes (DEGs) associated with bolting and flowering in radish (Raphanus sativus L.). Front Plant Sci. 2016;7:682.

8. Jeong YM, Kim N, Ahn BO, Oh M, Chung WH, Chung H, Jeong S, Lim KB, Hwang YJ, Kim GB. Elucidating the triplicated ancestral genome structure of radish based on chromosome-level comparison with the brassica genomes. Theor Appl Genet. 2016;129(7):1-16.

9. Huala E, Dickerman AW, Garcia-Hernandez M, Weems D, Reiser L, LaFond F, Hanley D, Kiphart D, Zhuang M, Huang W. The Arabidopsis information resource (TAIR): a comprehensive database and web-based information retrieval, analysis, and visualization system for a model plant. Nucleic Acids Res. 2001;29(1):102-5.

10. Cheng F, Liu S, Wu J, Fang L, Sun S, Liu B, Li P, Hua W, Wang XBRAD. The genetics and genomics database for brassica plants. BMC Plant Biol. 2011; 11(1):136.

11. Zhang X, Yue Z, Mei S, Qiu Y, Yang X, Chen X, et al. A de novo genome of a Chinese radish cultivar. Hortic. Plant J. 2015;1(3):155-64.

12. Parra G, Bradnam K, Korf ICEGMA. A pipeline to accurately annotate core genes in eukaryotic genomes. Bioinformatics. 2007;23(9):1061-7.

13. Cheng F, Wu J, Fang L, Wang X. Syntenic gene analysis between Brassica Rapa and other Brassicaceae species. Front Plant Sci. 2012;3(198):198.

14. Larkin MA, Blackshields G, Brown N, Chenna R, McGettigan PA, McWilliam H, Valentin F, Wallace IM, Wilm A, Lopez R, Clustal W, Clustal X. Version 2.0. Bioinformatics. 2007:23(21):2947-8.

15. Tamura K, Stecher G, Peterson D, Filipski A, Kumar S. MEGA6: molecular evolutionary genetics analysis version 6.0. Mol Biol Evol. 2013;30(12):2725-9.

16. Jiangtao C, Yingzhen K, Qian W, Yuhe S, Daping G, Jing L, Guanshan L. MapGene2Chrom, a tool to draw gene physical map based on Perl and SVG languages. Hereditas. 2015;37(1)

17. Trapnell C, Roberts A, Goff L, Pertea G, Kim D, Kelley DR, Pimentel H, Salzberg SL, Rinn JL, Pachter L. Differential gene and transcript expression analysis of RNA-seq experiments with TopHat and cufflinks. Nat Protoc. 2012:7(3):562-78

18. Kim D, Pertea G, Trapnell C, Pimentel H, Kelley R, Salzberg SL. TopHat2: accurate alignment of transcriptomes in the presence of insertions, deletions and gene fusions. Genome Biol. 2013;14(4):R36.
19. Kolde R. Pheatmap: pretty heatmaps. R package version. 2012;61

20. Edgar RCMUSCLE. Multiple sequence alignment with high accuracy and high throughput. Nucleic Acids Res. 2004;32(3):1792-7.

21. Li WH, CI W, Luo CCA. New method for estimating synonymous and nonsynonymous rates of nucleotide substitution considering the relative likelihood of nucleotide and codon changes. Mol Biol Evol. 1985;2(2):150-74.

22. Wang D, Zhang Y, Zhang Z, Zhu J, Yu J. KaKs_Calculator 2.0: a toolkit incorporating gamma-series methods and sliding window strategies. Genom, proteom. \& bioinform. 2010;8(1):77-80.

23. Liu S, Liu Y, Yang X, Tong C, Edwards D, Parkin IA, Zhao M, Ma J, Yu J, Huang S. The Brassica Oleracea genome reveals the asymmetrical evolution of polyploid genomes. Nat Commun. 2014;5(3930):3930-30.

24. Wang $X$, Wang $H$, Wang J, Sun $R$, Wu J, Liu S, Bai Y, Mun J-H, Bancroft I, Cheng $F$. The genome of the mesopolyploid crop species Brassica Rapa. Nat Genet. 2011:43(10):1035-9.

25. Lou P, Wu J, Cheng F, Cressman LG, Wang XW, CR MC. Preferential retention of circadian clock genes during diploidization following whole genome triplication in Brassica rapa. Plant cell. 2012;24(6):2415-26.

26. Wu F, Hanzawa Y. Photoperiodic control of flowering in plants. In: Pessarakli M, editor. Handbook of plant and crop physiology. 3rd ed. BocaRaton, Florida: CRC press; 2014. p. 129-39.

27. Baudry A, Ito S, Song YH, Strait AA, Kiba T, Lu S, Henriques R, Pruneda Paz JL, Chua NH, Tobin EM. F-Box proteins FKF1 and LKP2 act in concert with ZEITLUPE to control Arabidopsis clock progression. Plant Cell. 2010;22(3):606-22.

28. Fornara F, Panigrahi KC, Gissot L, Sauerbrunn N, Rühl M, Jarillo JA, Coupland G, Arabidopsis DOF. Transcription factors act redundantly to reduce CONSTANS expression and are essential for a photoperiodic flowering response. Dev Cell. 2009:17(1):75-86.

29. Harmer SL. The circadian system in higher plants. Annu Rev Plant Biol. 2009. 60:357-77.

30. Sawa M, Nusinow DA, Kay SA, Imaizumi T. FKF1 and GIGANTEA complex formation is required for day-length measurement in Arabidopsis. Science. 2007;318(5848):261-5.

31. Chen M, Chory J, Fankhauser C, et al. Annu Rev Genet. 2004:38:87-117. 30.

32. Sung S, He Y, Eshoo TW, Tamada Y, Johnson L, Nakahigashi K, Goto K, Jacobsen SE, Amasino RM. Epigenetic maintenance of the vernalized state in Arabidopsis thaliana requires LIKE HETEROCHROMATIN PROTEIN 1. Nat Genet. 2006:38(6):706-10

33. Srikanth A, Schmid M. Regulation of flowering time: all roads lead to Rome. Cell Mol Life Sci. 2011:68(12):2013-37.

34. Kim DH, Doyle MR, Sung S, Amasino RM. Vernalization: winter and the timing of flowering in plants. Annu Rev Cell Dev B. 2009;25:277-99.

35. Levy YY, Mesnage S, Mylne JS, Gendall AR, Dean C. Multiple roles of Arabidopsis VRN1 in vernalization and flowering time control. Science. 2002; 297(5579):243-6.

36. Sung S, Amasino RM. Vernalization and epigenetics: how plants remember winter. Curr Opin Plant Bio. 2004:7(1):4-10.

37. Gendall AR, Levy YY, Wilson A, Dean C. The VERNALIZATION 2 gene mediates the epigenetic regulation of vernalization in Arabidopsis. Cell. 2001;107(4):525-35.

38. Geraldo N, Bäurle I, Kidou S, Hu X, Dean CFRIGIDA. Delays flowering in Arabidopsis via a cotranscriptional mechanism involving direct interaction with the nuclear cap-binding complex. Plant Physiol. 2009:150(3):1611-8.

39. Schönrock N, Bouveret R, Leroy O, Borghi L, Köhler C, Gruissem W, Hennig L. Polycomb-group proteins repressthe floral activator AGL19 in the FLCindependent vernalization pathway. Genes Dev. 2006;20(12):1667-78.

40. Simpson GG. The autonomous pathway: epigenetic and post-transcriptional gene regulation in the control of Arabidopsis flowering time. Curr Opin Plant Bio. 2004;7(5):570-4.

41. Eriksson S, Böhlenius H, Moritz T, Nilsson O. GA4 is the active gibberellin in the regulation of LEAFY transcription and Arabidopsis floral initiation. Plant Cell. 2006:18(9):2172-81.

42. Mouradov A, Cremer F, Coupland G. Control of flowering time interacting pathways as a basis for diversity. Plant Cell. 2002;14:S111-30.

43. Silverstone AL, Mak PYA, Martinez EC, Sun T. The new RGA locus encodes a negative regulator of gibberellin response in Arabidopsis thaliana. Genetics. 1997;146(3):1087.

44. Parcy F. Flowering: a time for integration. Int J Dev Biol. 2005:49(5/6):585.

45. Jung WY, Park HJ, Lee A, Lee SS, Kim YS, Cho HS. Identification of floweringrelated genes responsible for differences in bolting time between two radish inbred lines. Front Plant Sci. 2016;7:1844. 
46. Thomas BC, Pedersen B, Freeling M. Following tetraploidy in an Arabidopsis ancestor, genes were removed preferentially from one homeolog leaving clusters enriched in dose-sensitive genes. Genome Res. 2006;16(7):934-46.

47. Force A, Lynch M, Pickett FB, Amores A, Yan Y, Postlethwait J. Preservation of duplicate genes by complementary, degenerative mutations. Genetics. 1999;151(4):1531-45.

48. Erwin JE, Warner RM, Smith AG. Vernalization, photoperiod and GA3 interact to affect flowering of Japanese radish (Raphanus sativus Chinese radish jumbo scarlet). Physiol Plantarum. 2002;115(2):298-302.

49. Freeling M. Bias in plant gene content following different sorts of duplication: tandem, whole-genome, segmental, or by transposition. Annu Rev Plant Biol. 2009;60:433-53.

50. Freeling $M$, Volff JN. The evolutionary position of subfunctionalization, downgraded. Genome Dynam. 2008;4:25.

51. Birchler JA, Veitia RA. The gene balance hypothesis: from classical genetics to modern genomics. Plant Cell. 2007;19(2):395-402.

52. Yi G, Park H, Kim J-S, Chae WB, Park S, Huh JH. Identification of three FLOWERING LOCUS C genes responsible for vernalization response in radish (Raphanus Sativus L.). Hortic Environ Biote. 2014;55(6):548-56.

53. Michaels SD, Amasino RM. Loss of FLOWERING LOCUS C activity eliminates the late-flowering phenotype of FRIGIDA and autonomous pathway mutations but not responsiveness to vernalization. Plant Cell. 2001;13(4):935-41.

54. Blümel M, Dally N, Jung C. Flowering time regulation in crops-what did we learn from Arabidopsis? Curr Opin Biotech. 2015;32:121-9.

55. Ashworth MB, Walsh MJ, Flower KC, et al. Directional selection for flowering time leads to adaptive evolution in Raphanus raphanistrum (wild radish). Evol Appl. 2016;9(4):619-29.

56. Jang S, Marchal V, Panigrahi KC, et al. Arabidopsis COP1 shapes the temporal pattern of $\mathrm{CO}$ accumulation conferring a photoperiodic flowering response. EMBO J. 2008;27(8):1277-88.

57. Liu L, Zhang Y, Li Q, et al. COP1-mediated ubiquitination of CONSTANS is implicated in cryptochrome regulation of flowering in Arabidopsis. Plant Cell. 2008:20(2):292-306.

\section{Submit your next manuscript to BioMed Central and we will help you at every step:}

- We accept pre-submission inquiries

- Our selector tool helps you to find the most relevant journal

- We provide round the clock customer support

- Convenient online submission

- Thorough peer review

- Inclusion in PubMed and all major indexing services

- Maximum visibility for your research

Submit your manuscript at www.biomedcentral.com/submit 